Research Article

\title{
Circular RNA hsa_circ_0006117 Facilitates Pancreatic Cancer Progression by Regulating the miR-96-5p/KRAS/MAPK Signaling Pathway
}

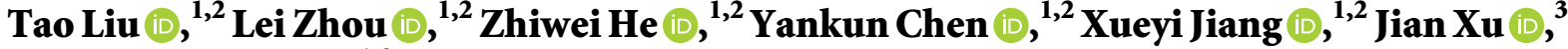 \\ and Jianxin Jiang $\mathbb{1}^{1,3}$ \\ ${ }^{1}$ School of Clinical Medicine, Guizhou Medical University, Guiyang 550000, China \\ ${ }^{2}$ Department of Hepatic-Biliary-Pancreatic Surgery, The Affiliated Hospital of Guizhou Medical University, \\ Guiyang 550000, China \\ ${ }^{3}$ Department of Hepatobiliary Surgery, Renmin Hospital of Wuhan University, Wuhan 430060, China
}

Correspondence should be addressed to Jianxin Jiang; rm002979@whu.edu.cn

Received 10 June 2021; Revised 5 August 2021; Accepted 17 August 2021; Published 3 September 2021

Academic Editor: Yuan Seng Wu

Copyright $\odot 2021$ Tao Liu et al. This is an open access article distributed under the Creative Commons Attribution License, which permits unrestricted use, distribution, and reproduction in any medium, provided the original work is properly cited.

Circular RNAs (circRNAs) play key roles in many malignant tumors, including pancreatic cancer (PC); however, whether circular RNA hsa_circ_0006117, a newly identified circRNA, has a role in PC has not been investigated. Here, in order to elucidate the role and potential molecular mechanisms of circRNAs, we utilized bioinformatic tolls to screen the differentially expressed circRNAs in PC. Subsequently, circular RNA hsa_circ_0006117 was identified as being highly expressed in PC tissues in a screen of two GEO datasets, which was further verified in PC cell lines and tissues. Then, its molecular characteristics were investigated using methods such as Sanger sequencing and fluorescence in situ hybridization (FISH). Functional experiments subsequently indicated that circular RNA hsa_circ_0006117 facilitated the malignant behaviors of PC cells, prompting that it plays an oncogenic role in PC. Moreover, we found that circular RNA hsa_circ_0006117 exerts its PC-promoting effects via activating the KRAS/mitogenactivated protein kinase (MAPK) signaling pathway. Through bioinformatics exploration and dual-luciferase reporter assays, miR-96-5p was identified as a downstream target of circular RNA hsa_circ_0006117. A series of assays confirmed that circular RNA hsa_circ_0006117 acted as a miR-96-5p sponge, thereby promoting the malignant features of PC in a miR-96-5p/KRAS axisdependent manner. Taken together, our study indicated, for the first time, that the specifically highly expressed circular RNA hsa_circ_0006117 facilitates PC progression via the modulation of the miR-96-5p/KRAS/MAPK signaling pathway and might be a hopeful therapeutic target for PC.

\section{Introduction}

Pancreatic cancer (PC) is a leading cause of cancer-related deaths worldwide among all cancers with the lowest fiveyear overall survival rate (10\%) [1]. Although surgical resection, neoadjuvant therapies, and comprehensive targeted treatment have improved the treatment options, early metastasis and invasion, combined with the lack of effective and precise targeted therapies, still limit the prognosis of PC patients [2-4]. Indeed, a severely poor prognosis with overall survival of just $10-16$ months is usually related to patients with locally advanced or unresectable PC [5]. This highlights the urgency in elucidating the molecular regulatory network in PC and searching for new or more effective therapeutic targets for treating disease.

Increasing evidence suggests that noncoding RNAs have widely participated in PC development [6-8]. Circular RNAs (circRNAs) are a sort of noncoding RNA stemmed from the back-splicing of precursor messenger RNAs (mRNAs). They have a stable covalent circular structure and are resistant to digestion by RNase R [9]. Compared with their linear precursors, circRNAs have functions that are 
independent of their host genes [10], such as serving as microRNA (miRNA) sponges or protein scaffolds, interacting with RNA-binding proteins, regulating alternative splicing or transcription, and generating pseudogenes $[11,12]$. Emerging shreds of evidence have revealed that circRNAs act as miRNA sponges to affect downstream targets and play roles in various cancers, including PC $[13,14]$. Nevertheless, the roles of circRNAs in PC remain poorly understood.

Members of the rat sarcoma (RAS) oncogene family, including Kirsten-RAS (KRAS), Harvey-RAS (HRAS), and neuroblastoma-RAS (NRAS), exhibit the highest mutation frequency in human cancers, with associated mutations being identified in approximately $30 \%$ of all cancers [15]. $K R A S$ is a driver gene of many diseases and one of the most common and frequently mutated genes in PC $[16,17]$. KRAS oncogenic mutations lead to the continued activation of downstream molecules, and the KRAS/mitogen-activated protein kinase (MAPK) signaling pathway is strongly associated with the development of PC, both of which enhance the malignant potential of this cancer $[18,19]$. Meanwhile, KRAS is also involved in the regulation of noncoding RNAs in some cancers [20-22]. However, the association between circRNAs and KRAS in PC has not been explored, nor has the underlying regulatory relationship.

Here, circular RNA hsa_circ_0006117 with high expression in PC was reidentified from two circRNA microarrays from the Gene Expression Omnibus (GEO) database. Subsequent functional experiments illustrated that circular RNA hsa_circ_0006117 promoted the rapid development of PC cells. Moreover, our results suggested that circular RNA hsa_circ_0006117 could activate the MAPK signaling pathway by relieving the miR-96-5p-mediated posttranscriptional suppression of KRAS. Taken together, we found that circular RNA hsa_circ_0006117 adsorbed miR-96-5p and acted in an axis-dependent regulation of miR-96-5p/ KRAS/MAPK, thereby facilitating proliferation, migration, and invasion in PC. Circular RNA hsa_circ_0006117 has the potential to represent a promising target for PC therapy.

\section{Materials and Methods}

2.1. Acquisition of Gene Expression and Identification of Differentially Expressed circRNAs (DECs). Using the keywords "circular RNA," "pancreatic cancer" or "pancreatic ductal adenocarcinoma," the expression profile of circular RNAs in PC were searched from the GEO (https://www.ncbi. nlm.nih.gov/geo/) database. The microarray datasets GSE69362 and GSE79634 were included and downloaded for screening potential DECs. After identifying DECs using the R "limma" package, Venn diagram analysis (http://bioinfor matics.psb.ugent.be/webtools/Venn/) was performed to overlap and focus candidate DECs.

2.2. Tissue Specimens and Cell Culture. We collected fresh pancreatic tissue specimens from 20 patients who had undergone pancreaticoduodenectomy or distal pancreatectomy at the Affiliated Hospital of Guizhou Medical
University, China. A pathological evaluation was employed to confirm the presence of PC. All patients with PC provided informed consent. This project was approved by the Ethics Committee of Guizhou Medical University (Approval Number: 2021 No. 129). Normal human pancreatic ductal epithelial cell (HPDE) was purchased from Beijing North Carolina Chuanglian Biotechnology Research Institute (Beijing, China). All PC cell lines were purchased from American Type Culture Collection (Manassas, VA, USA), including PANC-1, MIA PaCa-2, AsPC-1, BxPC-3, and SW1990. The cells were cultured in Dulbecco's modified Eagle's medium (DMEM) (Gibco, Waltham, USA) or Roswell Park Memorial Institute (RPMI) medium (Gibco) supplemented with $10 \%$ fetal bovine serum (Gibco).

2.3. RT-qPCR, Agarose Gel Electrophoresis, and Sanger Sequencing. We used an RNA-easy Isolation Reagent (Vazyme, Nanjing, China) to extract RNA based on its protocol. Real-time quantitative reverse transcription PCR (RT-qPCR) was performed using cDNA Synthesis Kit (Vazyme) and qPCR Probe Kit (Vazyme). Relative mRNA expression was analyzed with the $2^{-\Delta \Delta C T}$ method and standardized to those of the appropriate internal references. All primers are displayed in Table 1. Divergent primers were used for PCR amplification of reverse-transcribed cDNA and $2 \%$ agarose gel was selected for agarose gel electrophoresis. Then, agarose gel was used for Sanger sequencing with the assistance of Cloud-Seq Biotech (Shanghai, China).

2.4. RNase R Assay and Actinomycin D Treatment. Total RNA $(2 \mu \mathrm{g})$ was incubated with or without $3 \mathrm{U} / \mu \mathrm{g}$ RNase $\mathrm{R}$ (Epicentre, Madison, WI, USA) at $37^{\circ} \mathrm{C}$ for 30 minutes. Then, RT-qPCR was used for analyzing the abundance of circular RNA hsa_circ_0006117 and its parent gene protein tyrosine phosphatase receptor type A (PTPRA). Meanwhile, PC cells were treated with $2 \mu \mathrm{g} / \mathrm{mL}$ actinomycin D (SigmaAldrich, St Louis, MO, USA) or dimethyl sulfoxide (DMSO) (Sigma-Aldrich) for 12 hours. After harvesting the cells, the stability of circular RNA hsa_circ_0006117 and linear RNA PTPRA was tested by RT-qPCR.

2.5. Separation of Cytoplasmic and Nuclear Fractions. All subcellular RNA components of PC cells were isolated with a Cytoplasmic \& Nuclear RNA Purification Kit (Norgen Biotek, Thorold, ON, Canada) based on the protocol. While the cytoplasmic/nuclear RNA ratio was assessed by RT-qPCR, U6 was used as a positive reference for nuclear RNA and GAPDH as a positive reference for cytoplasmic RNA.

2.6. Fluorescence In Situ Hybridization (FISH). A FISH Kit (RiboBio, Guangzhou, China) was applied to visualize the subcellular localization of circRNA. PC cells were sowed in 24-well plates and reproduced until 60\%-70\% confluence. After fixation in $4 \%$ paraformaldehyde and permeabilization with TritonX-100, the cells were hatched with a prehybridization buffer for $30 \mathrm{~min}$ at $37^{\circ} \mathrm{C}$ and then with a Cy3-labeled circular RNA hsa_circ_0006117 probe (RiboBio) in 
TABLE 1: Sequences of primers and oligonucleotides.

\begin{tabular}{lcc}
\hline & Gene & \\
\hline Circular RNA hsa_circ_0006117 & Forward primer & Sequence $\left(5^{\prime}->3^{\prime}\right)$ \\
KRAS & Reverse primer & CCAGATAACCAGTTCACGGATG \\
& Forward primer & GCATCCATGCTTATCTGAAGG \\
PTPRA & Reverse primer & GCAAATAGAATTGATGGAGAAACC \\
& Forward primer & GCACCAACAGGAAATACCCA \\
GAPDH & Reverse primer & GAAGAGCTTATTGTCGTCTGCC \\
GRB2 & Forward primer & GAACGGGAAGCTCACTGG \\
& Reverse primer & GCCTGCTTCACCACCTTCT \\
IGF2BP2 & Forward primer & CTGGGTGGTGAAGTTCAATTCT \\
& Reverse primer & GTTCTATGTCCCGCAGGAATATC \\
RAP1A & Forward primer & AGCTAAGCGGGCATCAGTTTG \\
Oligonucleotides & Reverse primer & CCGCAGCGGGAAATCAATCT \\
miR-96-5p mimics & Forward primer & CGTGAGTACAAGCTAGTGGTCC \\
si-hsa_circ_0006117\#1 & Reverse primer & CCAGGATTTCGAGCATACACTG \\
si-hsa_circ_0006117\#2 & & UUUGGCACUAGCACAUUUUUGCU \\
si-hsa_circ_0006117\#3 & Sense strand & AGCAAAAAUGUGCUAGUGCCAAA \\
\hline
\end{tabular}

hybridization buffer overnight at $37^{\circ} \mathrm{C}$. Representative pictures were obtained using a fluorescence microscope (Olympus, Tokyo, Japan) at $\times 400$ magnification.

2.7. Western Blot. Dissolving PC cells with RIPA lysis buffer (Boster, Wuhan, China), 10\% SDS-PAGE was used to segregate proteins and then transferred them onto PVDF membranes (Millipore, MA, USA). After blocking with 5\% milk (total protein) or 5\% BSA (phosphorylated protein), the membranes were hatched with the corresponding primary antibodies and an HRP-conjugated secondary antibody. Immunoreactive protein bands were exposed with an ECL reagent (Boster) and quantified by Image Lab Software. The following commercially antibodies were used in this experiment: KRAS, GAPDH, phosphorylated-MEK1/2 (PMEK1/2), MEK1/2, phosphorylated-ERK1/2 (P-ERK1/2), and ERK (GAPDH and KRAS from ABclonal Technology, Wuhan, China; others from Cell Signaling Technology, Beverly, MA, USA).

2.8. Plasmids, Oligonucleotides, and Transfection. All oligonucleotides (miR-96-5p-inhibitor or mimics, small interfering RNA [siRNA]) targeting circular RNA hsa_circ_0006117, and their controls) were obtained from RiboBio (Guangzhou, China). The KRAS expression vector (pcDNA) was purchased from Vigenebio (Shandong, China). Opti-MEM and Lipofectamine 3000 (Invitrogen, Carlsbad, CA, USA) were performed to cotransfect constructs into PC cells. Then, short hairpin RNA (shRNA) targeting circular RNA hsa_circ_0006117 was generated by GeneChem (Shanghai, China).

2.9. Cell Counting Kit-8 (CCK-8) and Colony Formation Assay. PC cells transfected with 2000 cells/well were treated with CCK-8 reagent (CCK-8 kit; Bosterbio, China). At a specific time $(6 \mathrm{~h}, 24 \mathrm{~h}, 48 \mathrm{~h}, 72 \mathrm{~h}$, and $96 \mathrm{~h})$, a microplate reader (Thermo Fisher Scientific, Waltham, MA, USA) was used to record the absorbance at $450 \mathrm{~nm}$. Transfected PC cells were incubated with 800 cells/plate for 12 days for colony formation assay. After imaging, the number of clones was calculated and statistically analyzed.

2.10. Wound Healing Assay and Transwell Assay. After the transfected PC cells were planted, a $200 \mu \mathrm{L}$ pipette tip was applied to scratch the monolayer in transfected PC cells. Representative pictures were obtained with an inverted microscope (Olympus) at the appointed times ( 0 and $48 \mathrm{~h})$. The migration rate was normalized using the $0 \mathrm{~h}$ scratch area. At the same time, transwell assays was used to estimate the migratory and invasive capabilities of PC cells. The upper chamber was pretreated or not with $60 \mu \mathrm{L}$ Matrigel (Matrigel BD Biosciences, NY, USA) before transfection of $200 \mu \mathrm{L}$ serum-free PC cells $\left(5 \times 10^{4}\right.$ cells/well). And $600 \mu \mathrm{L}$ of $20 \%$ FBS medium was placed in the lower chamber. Migrating or invading cells were counted after $24-28 \mathrm{~h}$ of incubation. Representative pictures were captured with an inverted microscope (Olympus).

2.11. Circular RNA hsa_circ_0006117 Target Prediction and Bioinformatic Analysis. CircBANK (http://www.circbank. cn/searchCirc.html), TargetScan (http://www.targetscan. org/vert_72/), miRDB (http://mirdb.org/), and miRTarBase (http://miRTarBase.cuhk.edu.cn/) were performed to postulate circular RNA hsa_circ_0006117/miRNA binding and $\mathrm{mRNA} / \mathrm{miRNA}$ binding, respectively. Meanwhile, the "clusterProfiler" package in $\mathrm{R}$ was used for Kyoto Encyclopedia of Genes and Genomes (KEGG) enrichment analysis to ascertain the molecular regulatory network of circular RNA hsa_circ_0006117 using a threshold of $P$ value $<0.05$. 
2.12. Dual-Luciferase Reporter Assay. The circRNA-miRNA and miRNA-mRNA binding sites were predicted by StarBase (http://starbase.sysu.edu.cn/) and TargetScan. The circular RNA hsa_circ_0006117 sequence and that of the $3^{\prime}$ untranslated region ( $3^{\prime}$ UTR) of KRAS were amplified and separately inserted into the pmiR-RB-Report (Ribobio), termed as circRNA-WT or KRAS-WT. Meanwhile, the corresponding mutant sequences were also amplified and inserted into the same vector to generate circRNA-MUT and KRAS-MUT, respectively. After the cotransfection of these vectors (50 ng) and miR-96-5p control or miR-96-5p mimics $(50 \mathrm{~nm})$ into PC cells, a GLOMAX 96 spectrophotometer (Promega) and the Dual-Glo Luciferase Assay System (Promega, Madison, WI, USA) were applied to detect luciferase activity.

2.13. Statistical Analysis. SPSS version 26.0 (SPSS IBM, Armonk, NY, USA) was applied for statistical analysis. Independent sample Student's $t$-tests were used for comparisons between two groups, whereas one-way ANOVA was employed for comparisons of more than two groups. Pearson's correlation curve analysis was applied for assessing the correlations between different indicators. All tests were two-tailed, and a $P$ value of $<0.05$ suggested statistical significance.

\section{Results}

3.1. Identification and Characterization of Circular RNA hsa_circ_0006117. We identified potential DECs from GSE69362 and GSE79634. Venn diagram analysis showed that the overlapping circRNAs (circular RNA hsa_circ_0006117 \& circular RNA hsa_circ_0029634) in two datasets were both elevated in PC tissues (Figure 1(a) and Supplementary figures S1A and S1B). We further found that circular RNA hsa_circ_0006117 (Figure 1(b)) was differentially expressed between PC and paracancerous samples in each data set. Then, we concentrated on exploring circular RNA hsa_circ_0006117. RT-qPCR analysis indicated that circular RNA hsa_circ_0006117 (Figure 1(c)) was significantly upregulated in 20 pairs of PC tissues. Meanwhile, circular RNA hsa_circ_0006117 expression was upregulated in PC cells compared with that in HPDE cells (Figure $1(\mathrm{~d})$ ). In terms of the annotation in circBase (http://www.circbase.org/), we found that circular RNA hsa_circ_0006117 is derived from 5' of exon 8 to $3^{\prime}$ of exon 9 of PTPRA (Chr20: 2944917-2945848) by back-splicing (Figure 1(e), upper panel). Thus, we designed divergent and convergent primers to amplify the back-spliced (circular RNA hsa_circ_0006117) and linear products (PTPRA), respectively. Circular RNA hsa_circ_0006117 could only be amplified from cDNA obtained from MIA PaCa-2 cells using divergent primers (Figure 1(e) (lower panel), Figure 1(f)). Next, Sanger sequencing of PCR amplicons validated the back-splicing site of circular RNA hsa_circ_0006117 (Figure 1(g)). After that, the stability of circular RNA hsa_circ_0006117 in PC cells treated with RNase R or actinomycin D was analyzed. RT-qPCR results revealed that circular RNA hsa_circ_0006117 had strong tolerance to
RNase $\mathrm{R}$ digestion, whereas the linear form of PTPRA was rapidly degraded (Figure $1(\mathrm{~h})$ ). A stability assay using actinomycin D treatment also demonstrated that circular RNA hsa_circ_0006117 was substantially more stable than linear PTPRA in PC cells (Supplementary figure S1C). Furthermore, separation of cytoplasmic and nuclear fractions (Figure 1(i)) and FISH (Figure 1(j)) suggested that the subcellular localization of circular RNA hsa_circ_0006117 was mainly in the cytoplasm.

3.2. Circular RNA hsa_circ_0006117 Facilitated the Proliferation, Migration, and Invasion of PC Cells. A series of loss-offunction experiments were applied to illustrate the function of circular RNA hsa_circ_0006117 in PC. For this, we designed siRNAs targeting the unique back-splicing site of circular RNA hsa_circ_0006117 (Supplementary figure S2) and used RT-qPCR to confirm that transfection of these siRNAs reduced the expression of circular RNA hsa_circ_0006117, but not that of PTPRA (Figure 2(a)). Because circRNA-si\#1 and circRNA-si\#2 elicited the best results, they were used for subsequent experiments. The proliferation capacity was suppressed by the downregulation of circular RNA hsa_circ_0006117 in PC cells, which was confirmed by CCK-8 (Figure 2(b)) and colony formation (Figures 2(c) and 2(d)) assays. Moreover, both wound healing (Figure 2(e)) and transwell (Figures 2(f) and 2(g)) assays indicated that downregulating circular RNA hsa_circ_0006117 markedly weakened the invasiveness and migratory capability of PC cells.

\subsection{Circular RNA hsa_circ_0006117 Maintained the Malig-} nant Characteristics of $P C$ via Activating the MAPK Signaling Pathway. Studies have suggested that exon-derived circRNAs mainly exert their functions in tumor development by sponging miRNAs [12]. To explore whether circular RNA hsa_circ_0006117 functions as a miRNA sponge, we used bioinformatics databases, such as circBANK, miRDB, TargetScan, and miRTarBase, to predict the targets of circular RNA hsa_circ_0006117 within the competitive endogenous RNA network associated with this circRNA. Meanwhile, we found that the MAPK and RAS signaling pathways were the most likely downstream targets of circular RNA hsa_circ_0006117 in KEGG enrichment analysis (Figure 3(a)). Subsequently, combined with gene expression profile from The Cancer Genome Atlas (TCGA) database, we focused on genes involved in the MAPK signaling pathway and identified highly expressed KRAS, GRB2, IGF2BP2, and RAP1A in $\mathrm{PC}$ tissues as the most significantly enriched genes, implicating them as potential targets of circular RNA hsa_circ_0006117 (Figure 3(b) and Supplementary figures S3A-S3C). In addition, we found that KRAS reduced overall survival in patients with PC based on data from the Gene Expression Profiling Interactive Analysis (GEPIA) database (http://gepia2.cancer-pku.cn/\#index) (Figure 3(c)). Therefore, we further constructed shRNAs using the sequence of circRNA-si\#1 and circRNA-si\#2 and then transfected them into PC cells for follow-up experiments. Interestingly, the mRNA (Figure 3(d) and Supplementary figure S3D) and 


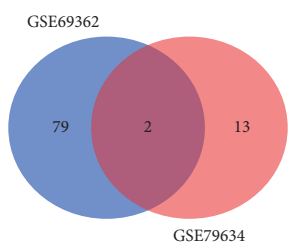

(a)

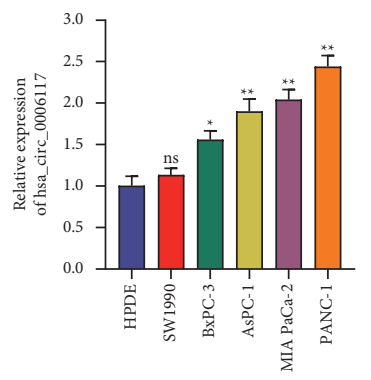

(d)

Splicing junction

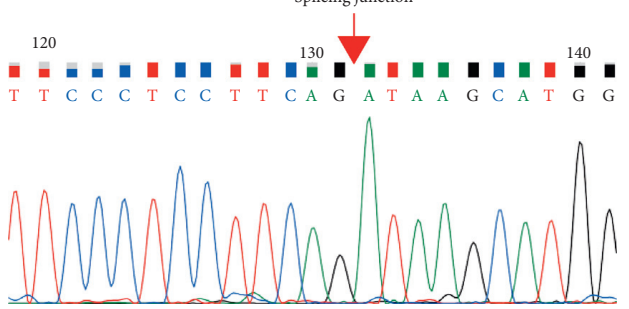

(g)

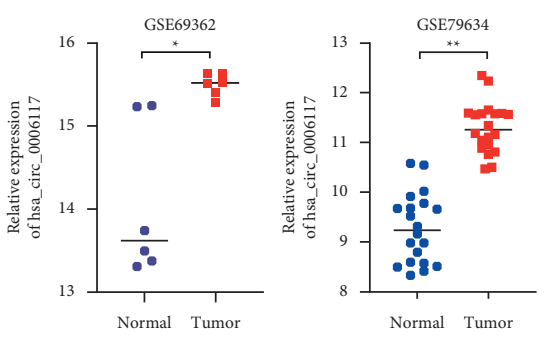

(b)
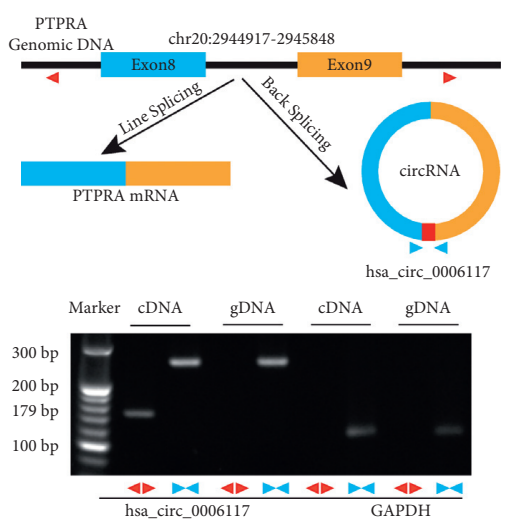

(e)

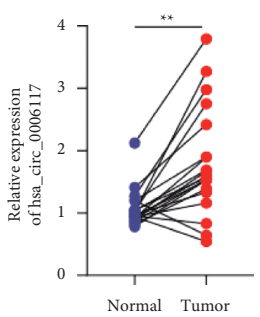

(c)

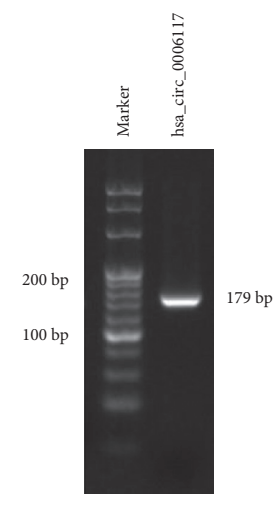

(f)

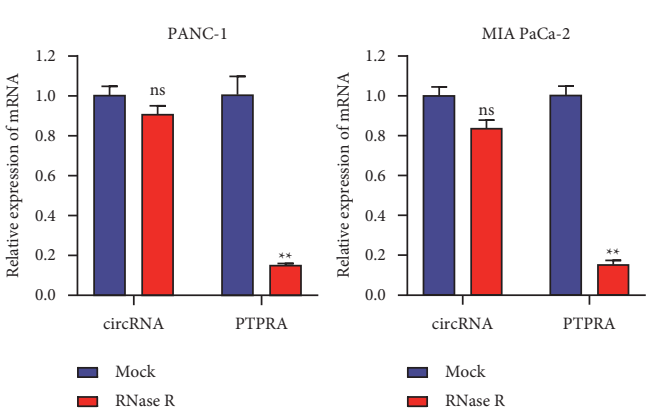

(h)
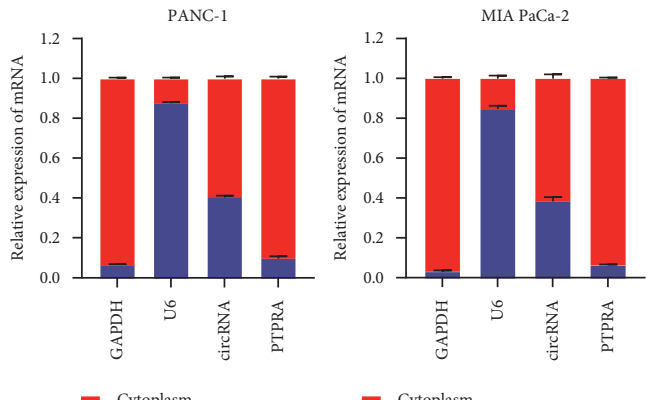

- Nucleus

- Cytoplasm

(i)

Figure 1: Continued. 


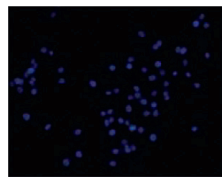

DAPI

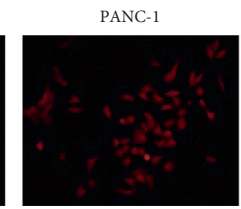

hsa_circ_0006117

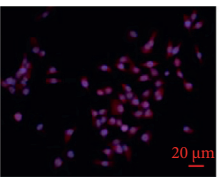

Merge

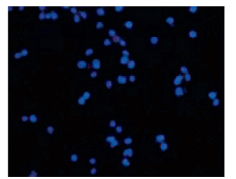

DAPI

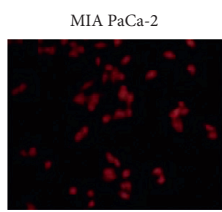

hsa_circ_0006117

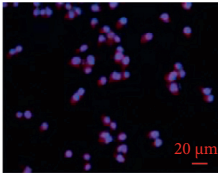

Merge

(j)

Figure 1: The identification and characterization of circular RNA hsa_circ_0006117. (a) Differentially expressed circRNAs that are upregulated in both GSE69362 and GSE79634 datasets. (b) Circular RNA hsa_circ_0006117 is differentially expressed between pancreatic cancer (PC) and paracancerous samples in GSE69362 and GSE79634. (c) The expression of circular RNA hsa_circ_0006117 in 20 pairs of matched PC tissues (tumor) and the corresponding paracarcinoma tissues (normal). (d) Circular RNA hsa_circ_0006117 expression in PC cell lines was tested by RT-qPCR. (e) Upper panel: schematic illustrations showing the genomic location of PTPRA and that of circular RNA hsa_circ_0006117 derived from a region $5^{\prime}$ of exon 8 to $3^{\prime}$ of exon 9 of the PTPRA locus. Lower panel: agarose gel electrophoresis analysis of circular RNA hsa_circ_0006117 and GAPDH in cDNA and genomic DNA (gDNA) derived from MIA PaCa-2 cells. (f) RT-PCR with divergent primers showed that circular RNA hsa_circ_0006117 was expressed in cDNA derived from MIA PaCa-2 cells. (g) The backsplicing junction (red arrow) associated with circular RNA hsa_circ_0006117 generation as identified by Sanger sequencing. (h) The relative mRNA expression of circular RNA hsa_circ_0006117 and PTPRA in PC cells after RNase R treatment. (i, j) The subcellular distribution of circular RNA hsa_circ_0006117 was ascertained by RT-qPCR (i) and fluorescence in situ hybridization (FISH) (j). GAPDH and U6 were used as reference indicators for the subcellular distribution of circular RNA hsa_circ_0006117 in the cytoplasm and nucleus, respectively. The circular RNA hsa_circ_0006117 probe was labeled with Cy3 (red) and the nucleus was counterstained with DAPI (blue). Representative images were obtained at $\times 400$ magnification (bars: $20 \mu \mathrm{m}$ ). All values were shown as means \pm SD, ns: not significant, ${ }^{*} P<0.05$, and ${ }^{* *} P<0.001$.

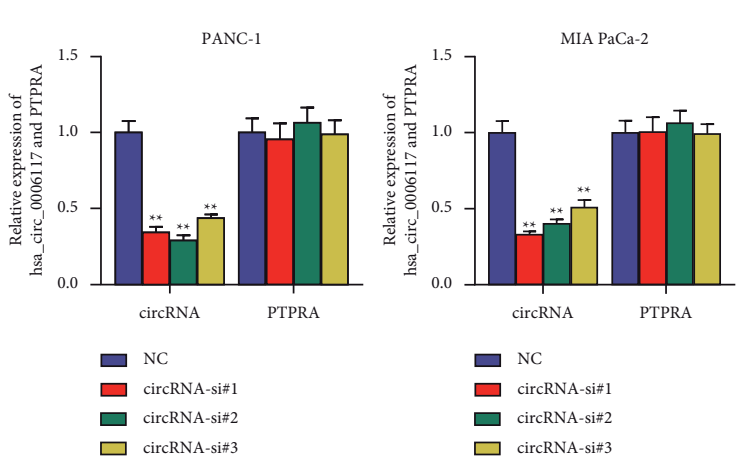

(a)

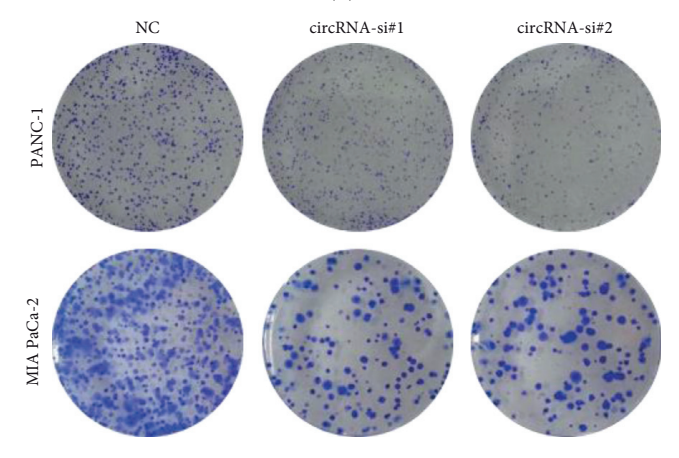

(c)
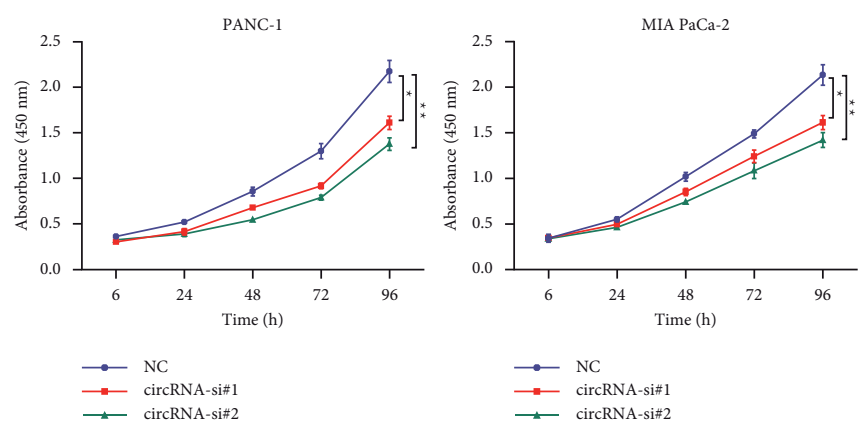

(b)
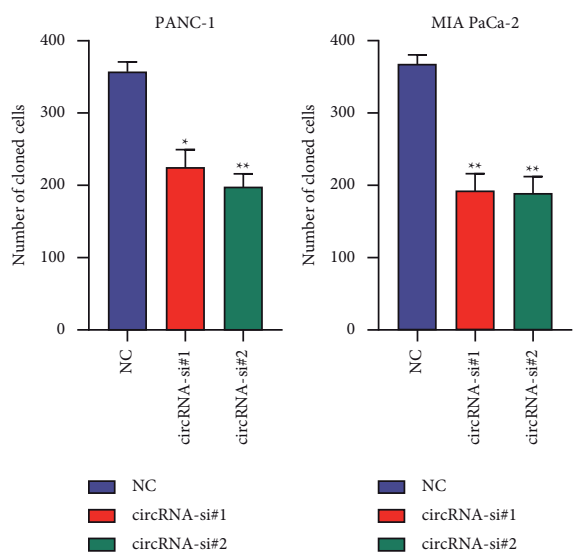

(d)

FIGURE 2: Continued. 

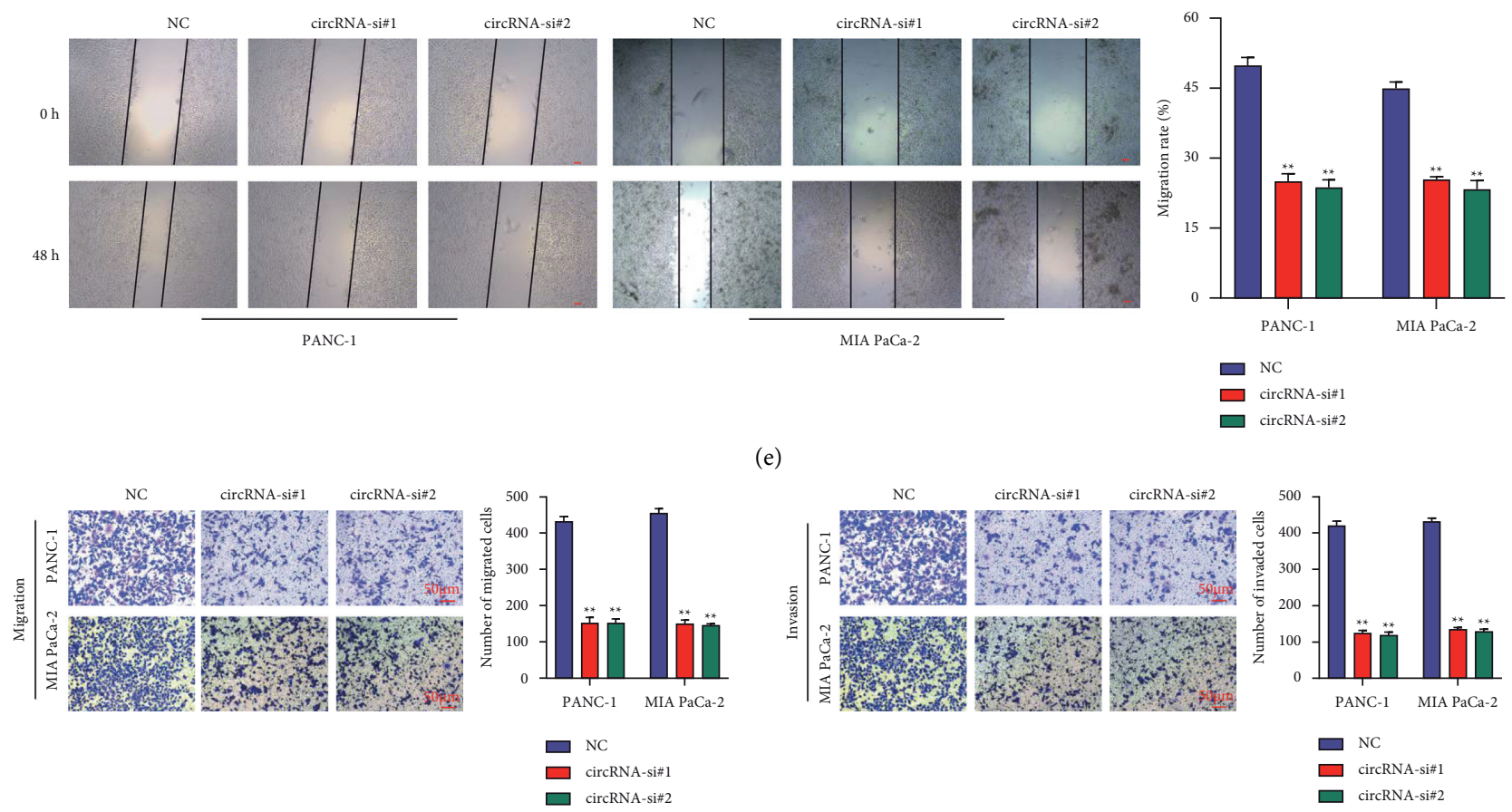

(e)

(f)

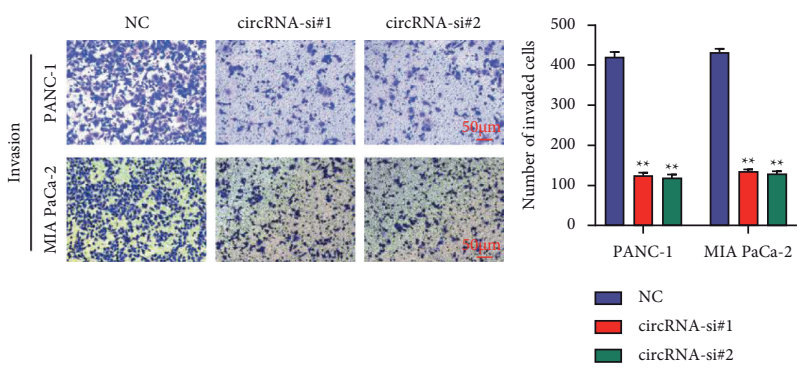

(g)

FiguRE 2: Circular RNA hsa_circ_0006117 facilitates the proliferation, migration, and invasion of pancreatic cancer (PC) cells. (a) The mRNA expression of circular RNA hsa_circ_0006117 and PTPRA in circular RNA hsa_circ_0006117-silenced PC cells. (b) Cell counting kit-8 (CCK-8) assays in circular RNA hsa_circ_0006117-silenced PC cells. (c, d) Colony formation assays in circular RNA hsa_circ_0006117-silenced PC cells. (e) Wound healing assays in circular RNA hsa_circ_0006117-silenced PC cells. Representative images were obtained at $\times 40$ magnification (bars: $100 \mu \mathrm{m}$ ). (f, g) Transwell migration (f) and invasion (g) assays in circular RNA hsa_circ_0006117silenced PC cells were applied to evaluate the invasiveness and migration capabilities of PC, respectively. Representative images were obtained at $\times 200$ magnification (bars: $50 \mu \mathrm{m}$ ). All values were shown as means \pm SD, ${ }^{*} P<0.05$, and ${ }^{* *} P<0.001$.

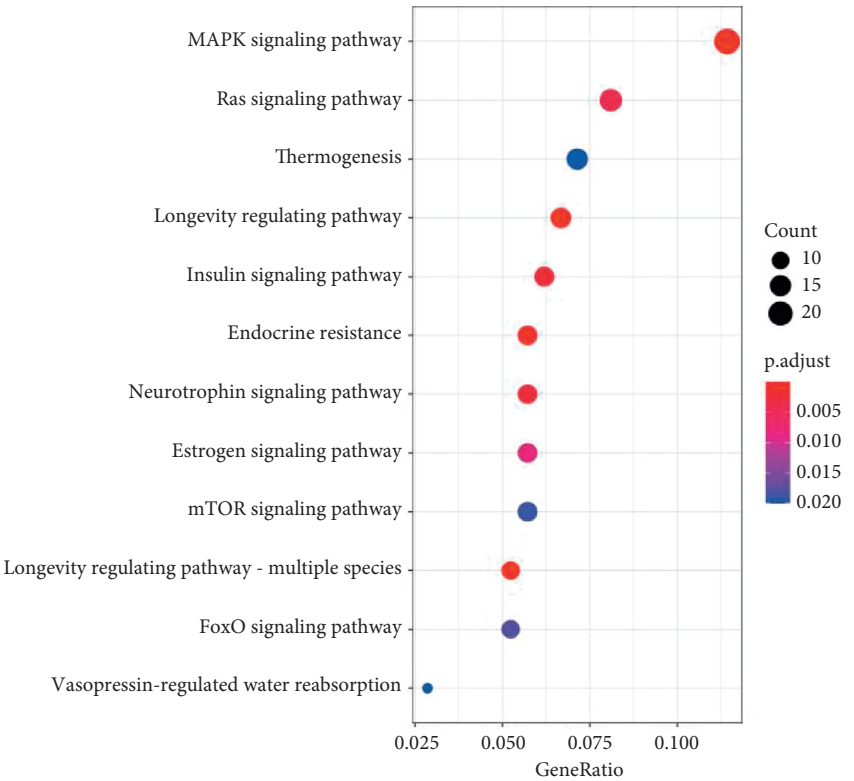

(a)

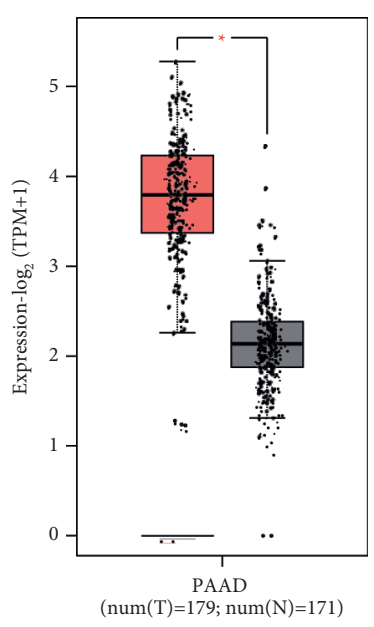

(b)

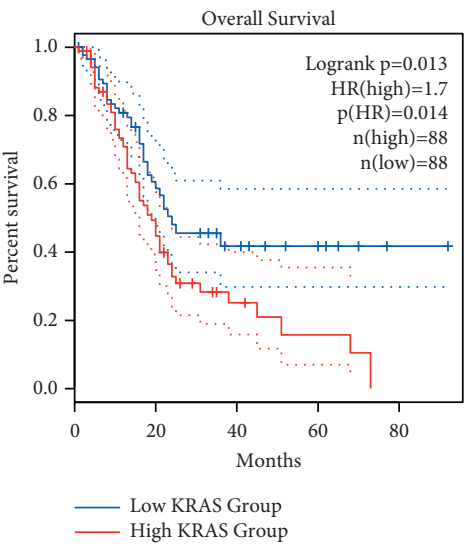

(c)

Figure 3: Continued. 


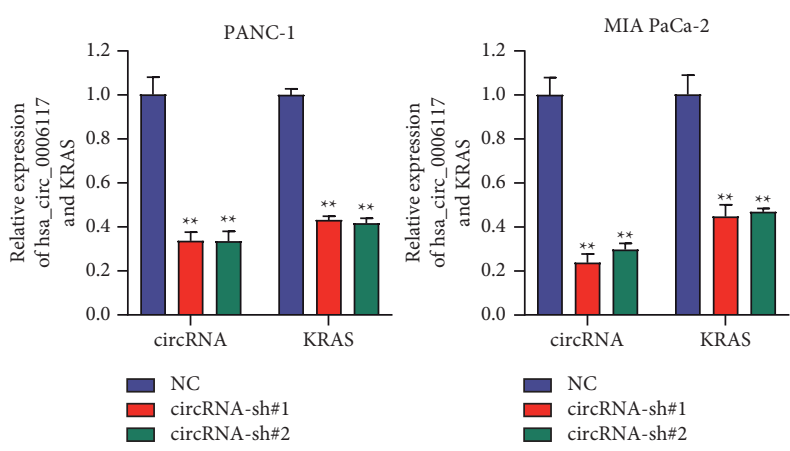

(d)

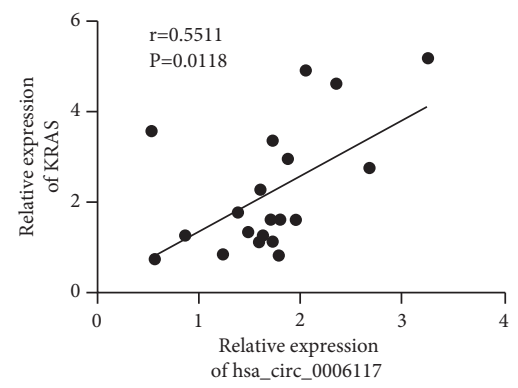

(f)

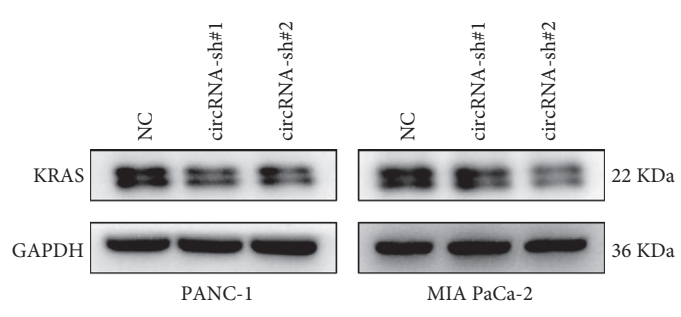

(e)

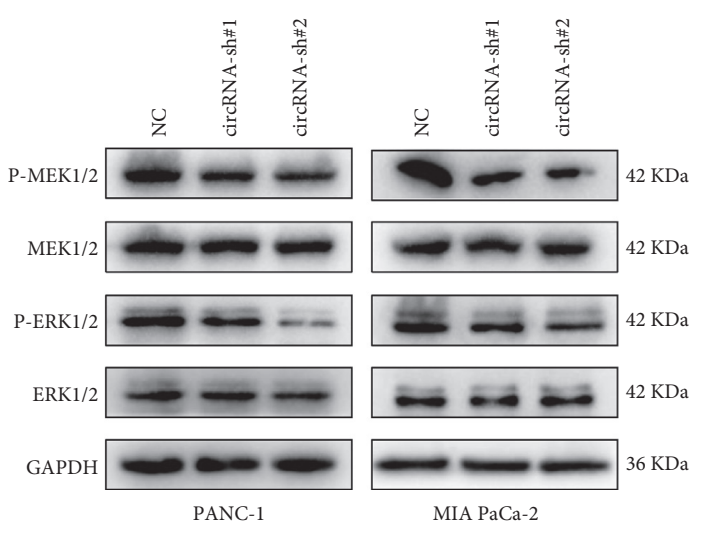

(g)

Figure 3: Circular RNA hsa_circ_0006117 maintains the malignant characteristics of pancreatic cancer (PC) via activating the MAPK signaling pathway. (a) The potential circular RNA hsa_circ_0006117-associated signaling pathways were identified by KEGG analysis in R. (b) KRAS is highly expressed in PC samples from the TCGA database, which was queried by the GEPIA database. (c) The overall survival data of PC patients obtained from the TCGA database; the dotted line indicates the 95\% confidence interval. (d, e) RT-qPCR and western blot assays in circular RNA hsa_circ_0006117-silenced PC cells were adopted to measure the mRNA (d) and protein content (e) of KRAS. (f) The correlation between circular RNA hsa_circ_0006117 and KRAS was estimated by Pearson's correlation curve analysis. (g) The expression of P-MEK1/2, MEK1/2, P-ERK1/2, and ERK1/2 were detected in circular RNA hsa_circ_0006117-silenced PC cells. All values were shown as means $\pm \mathrm{SD},{ }^{*} P<0.05$, and ${ }^{* *} P<0.001$.

protein (Figure 3(e)) expression of KRAS were both downregulated in PC cells transfected with shRNA targeting circular RNA hsa_circ_0006117. Moreover, a direct relationship between the expression of circular RNA hsa_circ_0006117 and KRAS in PC tissue samples was revealed by Pearson's correlation analysis (Figure 3(f)). It is well known that KRAS contributes to the upregulation of the MAPK signaling pathway, we hypothesized that circular RNA hsa_circ_0006117 was also involved in the influence on the MAPK signaling pathway. We verified that the protein levels of phosphorylated mitogen-activated extracellular signal-regulated kinase $1 / 2$ (P-MEK1/2) and phosphorylated extracellular signal-regulated kinases $1 / 2$ (P-ERK1/2) were lower in circular RNA hsa_circ_0006117-silenced PC cells than in the negative controls, whereas the total MEK $1 / 2$ and ERK $1 / 2$ protein level remained unchanged (Figure $3(\mathrm{~g})$ ).

3.4. The Circular RNA hsa_circ_0006117-Mediated Malignant Progression of PC Was Dependent on KRAS. To verify whether circular RNA hsa_circ_0006117 facilitates PC progression through regulating KRAS, we overexpressed KRAS in circular RNA hsa_circ_0006117-silenced PC cells to rescue the inhibitory effects of circRNA-sh\#2. Proliferative capacity assays revealed that ectopically expressed KRAS could partially rescue the effects induced by circular RNA hsa_circ_0006117 silencing and facilitated the growth of PC cells (Figures 4(a) and 4(b)). Similarly, wound healing (Figure 4(c)) and transwell (Figures 4(d) and 4(e)) assays demonstrated that KRAS overexpression partially rescued the circular RNA hsa_circ_0006117 knockdown-induced effects and reinforced the migration and invasion potential of $\mathrm{PC}$ cells. Furthermore, in PC cells where circular RNA hsa_circ_0006117 was knocked down, KRAS transfection partially restored the expression of P-MEK1/2 and P-ERK1/2 when the total MEK1/2 and ERK1/2 remained constant (Figure 4(f)).

3.5. The Circular RNA hsa_circ_0006117-Dependent KRAS Regulation in PC Progression Was Mediated by miR-96-5p. The above studies have shown that circular RNA hsa_circ_0006117 may play PC-promoting effects by sponging 

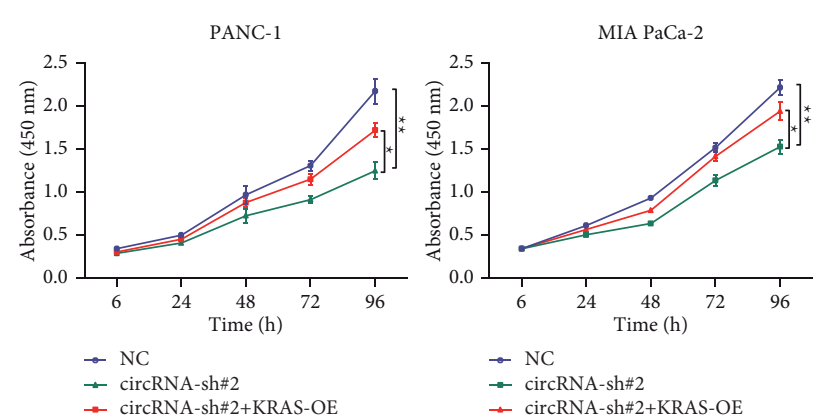

$\rightarrow \mathrm{NC}$

$\rightarrow$ circRNA-sh\#2

- circRNA-sh\#2+KRAS-OE

(a)

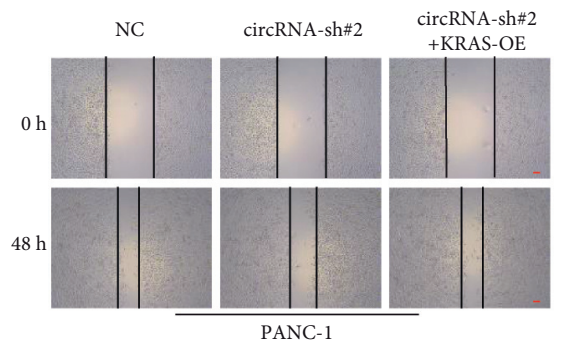

(d)

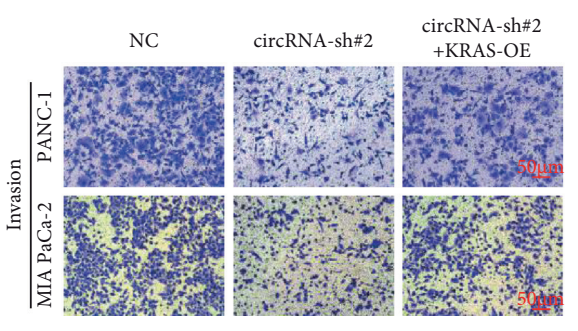

(e)

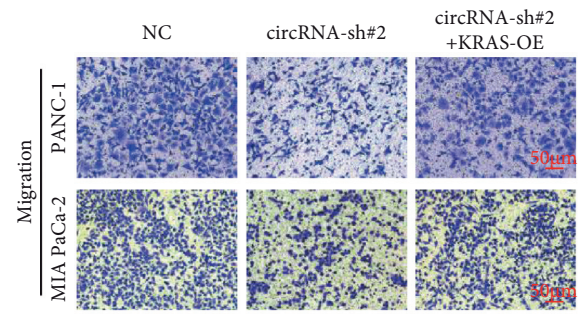

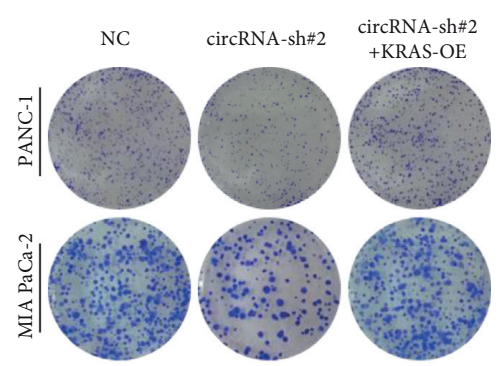

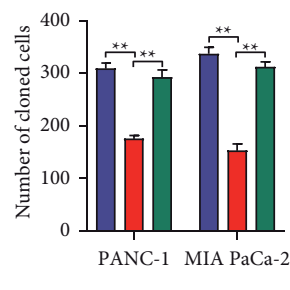

$\square \mathrm{NC}$

$\square$ circRNA-sh\#2

$\square$ circRNA-sh\#2+KRAS-OE

(b)

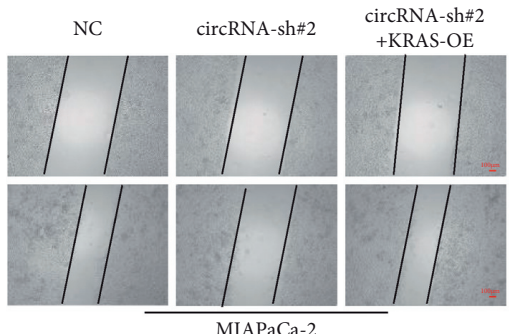

(c)
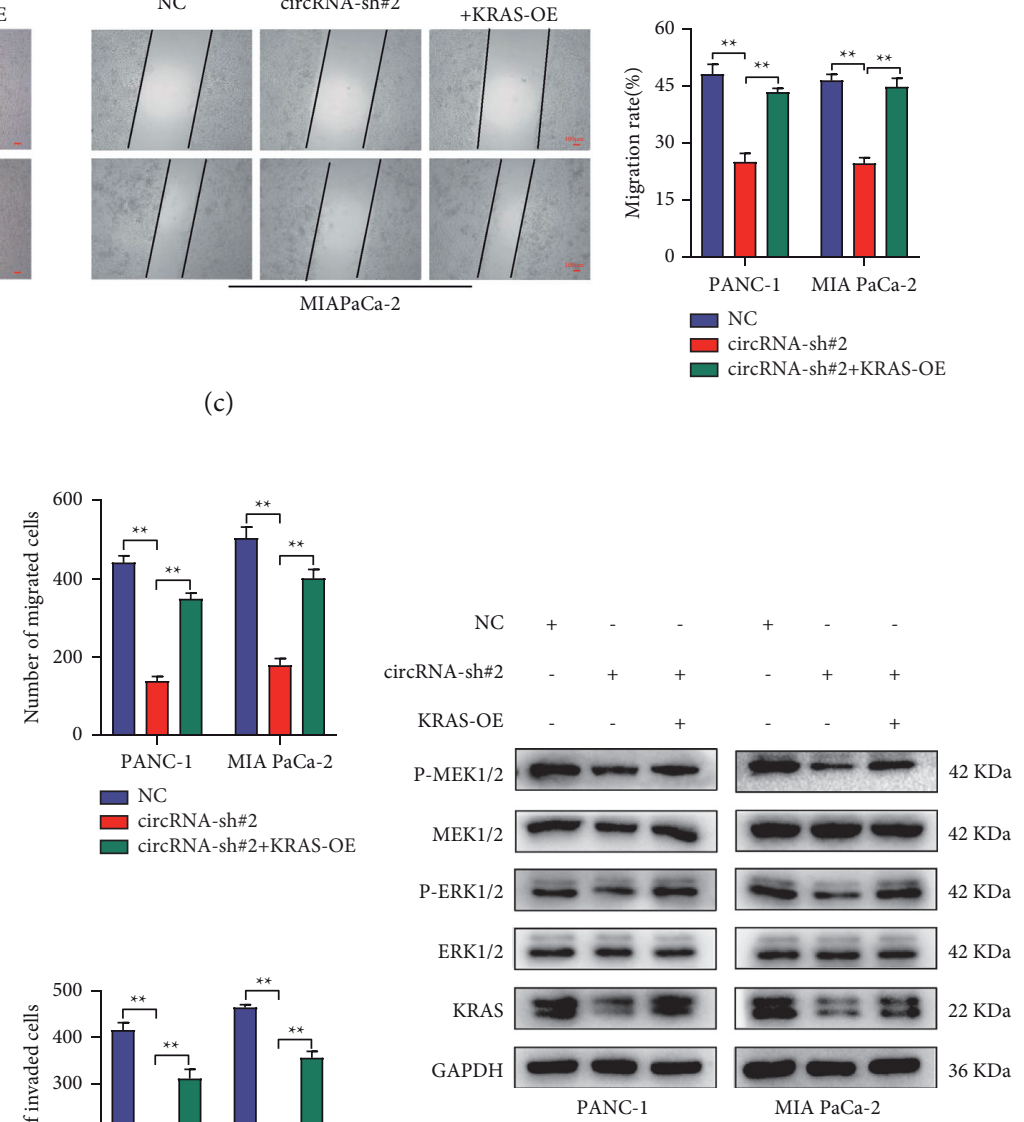

(f)

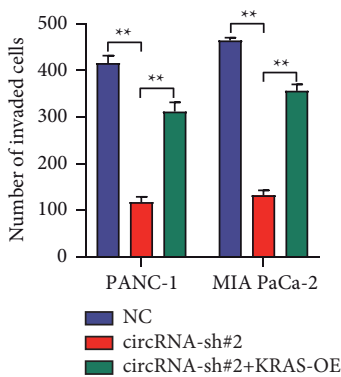

MIA PaCa-2

FIGURE 4: The circular RNA hsa_circ 0006117-mediated malignant progression of pancreatic cancer (PC) is KRAS-dependent. (a) The growth ability of PC cells was confirmed with cell counting kit-8 (CCK-8) assay in circular RNA hsa_circ_0006117-silenced and (or) KRAS-overexpressing PC cells. (b) The colony-forming ability of PC cells was evaluated by colony formation assay in circular RNA hsa_circ_0006117silenced and (or) KRAS-overexpressing PC cells. (c) The migratory capacity of PC cells was estimated by wound healing assay in circular RNA hsa_circ_0006117-silenced and (or) KRAS-overexpressing PC cells. Representative images were obtained at $\times 40$ magnification (bars: $100 \mu \mathrm{m}$ ). (d, e) The invasive and migratory capabilities of PC cells in rescue experiments were investigated by transwell migration (d) and invasion (e) assays, respectively. Representative images were obtained at $\times 200$ magnification (bars: $50 \mu \mathrm{m}$ ). (f) The expression of key proteins associated with the MAPK signaling pathway in rescue experiments. All values were shown as means $\pm \mathrm{SD},{ }^{*} P<0.05$, and ${ }^{* *} P<0.001$. 


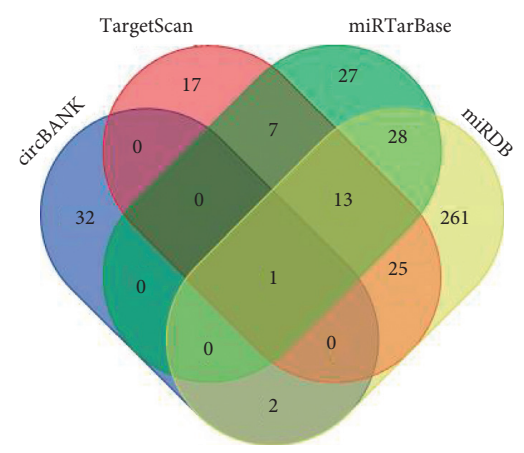

(a)

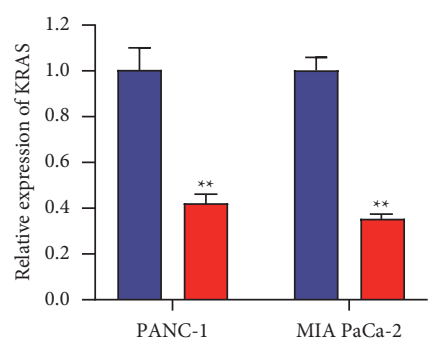

$\square$ miR-96-5p-control

$\square$ miR-96-5p-mimics

(d)
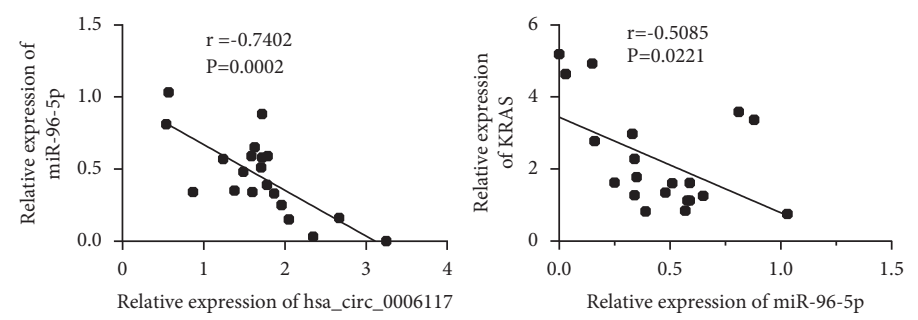

(f)
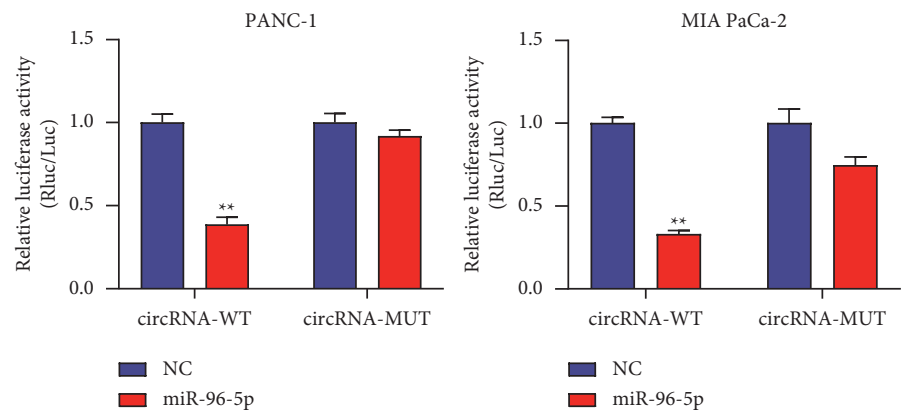

(h)
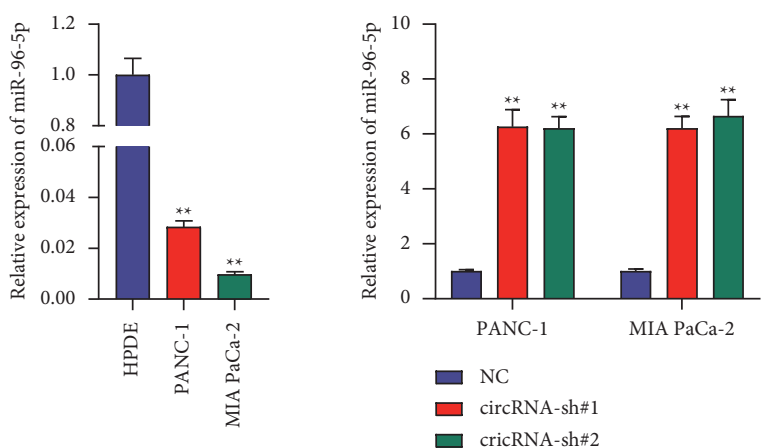

(b)

(c)

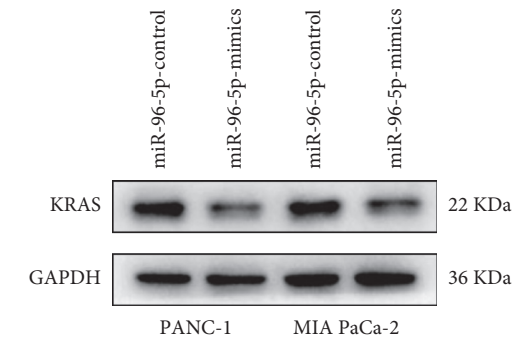

(e)

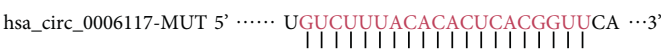

hsa_circ_0006117-WT 5 , …. UCUGAUAUGUGUCAGUGCCAACA $\cdots 3$, hsa_miR-96-5p $\quad \begin{gathered}\mid \\ 3\end{gathered}$

(g)

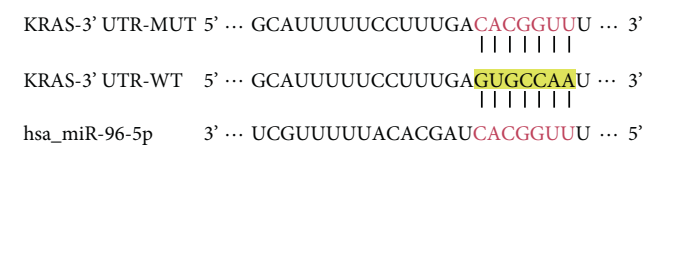

(i)

Figure 5: Continued. 

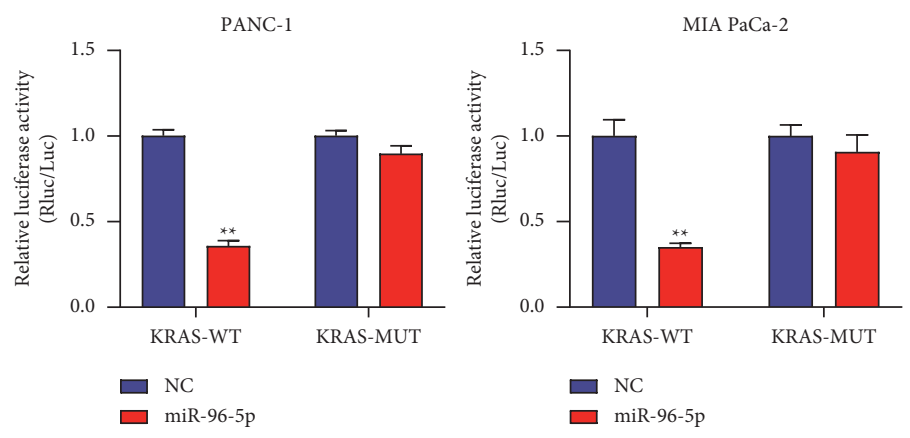

(j)

FIGURE 5: MiR-96-5p mediates the circular RNA hsa_circ_6117-associated regulation of KRAS in the progression of pancreatic cancer (PC). (a) A Venn diagram showing the overlapping miRNAs that simultaneously bind to circular RNA hsa_circ_0006117 and KRAS as predicted by circBANK, TargetScan, miRDB, and miRTarBase. (b) Compared with HPDE cells, RT-qPCR showing the mRNA content of miR-96-5p in PC cells. (c) The mRNA content of miR-96-5p was detected in circular RNA hsa_circ_0006117-silenced PC cells. (d, e) The mRNA (d) and protein content (e) of KRAS in miR-96-5p-overexpressing PC cells. (f) Pearson's correlation curve analysis provided a direct link between miR-96-5p and circular RNA hsa_circ_0006117 or KRAS. (g) A schematic diagram showing the putative conjugated site between circular RNA hsa_circ_0006117 and miR-96-5p. (h) The relative luciferase intensity in PC cells cotransfected with the miR-96-5p mimics or miR-96$5 \mathrm{p}$ control and circRNA-WT (containing the wild-type (WT) circular RNA hsa_circ_0006117 sequence) or circRNA-MUT (containing a mutated [MUT] circular RNA hsa_circ_0006117 sequence). Firefly luciferase (Luc) intensity was normalized to that of Renilla luciferase (Rluc). (i) A schematic diagram showing the putative conjugated site between miR-96-5p and the $3^{\prime}$ UTR of KRAS. (j) The relative luciferase intensity in PC cells cotransfected with the miR-96-5p control or miR-96-5p mimics and KRAS-WT (containing the wild-type (WT) miR96-5p-binding sequence) or KRAS-MUT (containing a mutated (MUT) miR-96-5p-binding sequence). Luc intensity was normalized to that of Rluc. All values were shown as means $\pm \mathrm{SD},{ }^{*} P<0.05$, and ${ }^{* *} P<0.001$.

miRNAs. Hence, we used bioinformatic analysis to explore which miRNAs can bind both circular RNA hsa_circ_0006117 and KRAS, and miR-96-5p was identified as a possible candidate (Figure 5(a)). RT-qPCR indicated that miR-96-5p was lower expressed in PC cells than in HPDE cells (Figure 5(b)). On the contrary, miR-96-5p content in PC cells transfected with circRNA-sh\#1 and circRNA-sh\#2 increased (Figure 5(c)), whereas the content of KRAS in PC cells transfected with miR-96-5p mimics reduced (Figures 5(d), and 5(e)). Furthermore, the data presented in Figure 5(f) showed that miR-96-5p content was inversely associated with the content of both circular RNA hsa_circ_0006117 and KRAS. These results suggested that circular RNA hsa_circ_0006117 and KRAS may share miR-96-5p binding sites. We subsequently generated pmiR-RB-Report ${ }^{\mathrm{TM}}$ constructs containing either circular RNA hsa_circ_0006117 or KRAS 3'UTR sequences and confirmed them by sequencing (Supplementary figures S4A-S4D). As expected, the luciferase intensity of PC cells cotransfected with miR-96-5p mimics and circRNA-WT was significantly weaker than that cotransfected with miR-96-5p mimics and circRNAMUT, prompting that miR-96-5p and circular RNA hsa_circ_0006117 have complementary binding sequences (Figures 5(g) and 5(h)). Meanwhile, the same complementary sequences were also present in the KRAS $3^{\prime}$ UTR (the predicted binding sites are displayed in Figure 5(i)). Subsequently, the results demonstrated that PC cells cotransfected with the miR-96-5p mimics KRASWT, but not KRAS-MUT, displayed reduced luciferase activity, suggesting that miR-96-5p interacted with KRAS by binding to sequences in its $3^{\prime}$ UTR (Figure $5(\mathrm{j})$ ).
3.6. Circular RNA hsa_circ_0006117 Facilitated PC Development by Adsorbing miR-96-5p. To investigate whether circular RNA hsa_circ_0006117 facilitates PC development by regulating miR-96-5p, in circular RNA hsa_circ_0006117-silenced PC cells, miR-96-5p-inhibitor was transfected or cotransfected for subsequent rescue experiments. Experiments reflecting proliferative capacity (Figures 6(a) and 6(b)) suggested that miR-96-5p-inhibitor facilitated the growth of PC cells, whereas cotransfection with miR-96-5p-inhibitor and circRNA-sh\#2 prevented the inhibition of circular RNA hsa_circ_0006117, thereby accelerating the growth of PC cells. Similarly, following rescue experiments, wound healing (Figures 6(c) and 6(d)) and transwell (Figures 6(e) and 6(f)) assays demonstrated that cotransfection with miR-96-5p-inhibitor could restore the loss of circular RNA hsa_circ_0006117 expression and promote PC migration and invasion. Moreover, in PC cells where circular RNA hsa_circ_0006117 was knocked down, miR-96-5p-inhibitor rescued the phosphorylation of MEK1/ 2 and ERK1/2, which were key activators of the MAPK signaling pathway (Figure 6(g)).

\section{Discussion}

PC displays highly invasive and metastatic characteristics $[23,24]$. Advances in the comprehensive systemic treatment of PC have not resulted in improvements in the prognosis of this cancer [25]. Numerous pieces of evidence have suggested that circRNAs have inspired the development of PC. A study by Li et al. [26] revealed that circ-IARS secreted by PC cells affected endothelial monolayer permeability, thereby facilitating PC invasion and metastasis. In addition, 

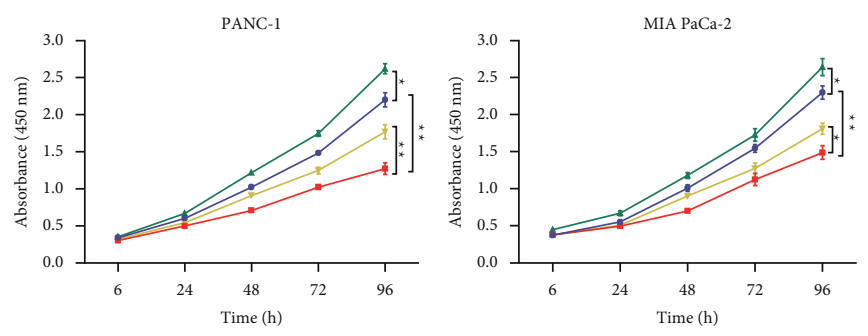

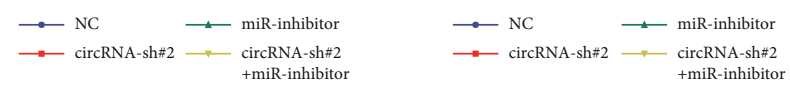

(a)

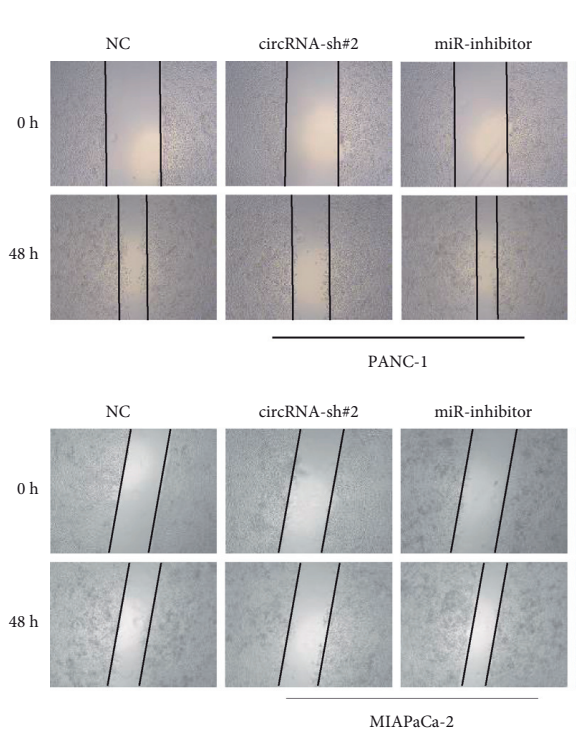

(c)
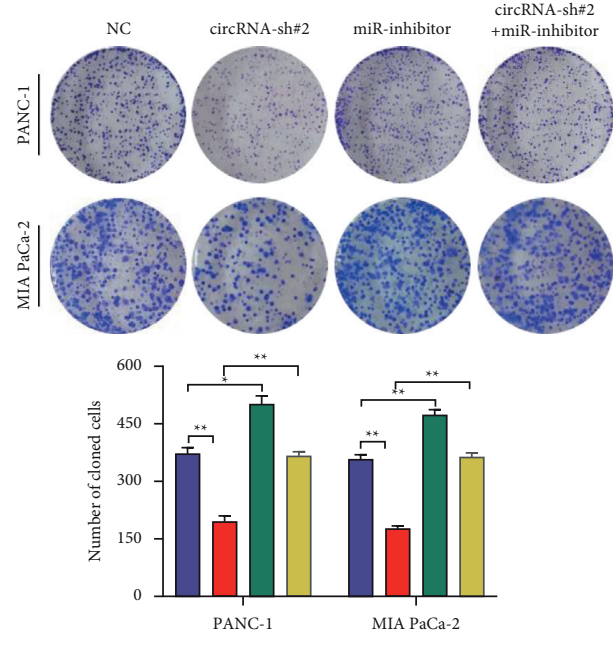

口 $\mathrm{NC}$ miR-inhibitor

$\square$ circRNA-sh\#2 $\square \begin{gathered}\text { circRNA-sh\#2+ } \\ \text { miR-inhibitor }\end{gathered}$

(b)

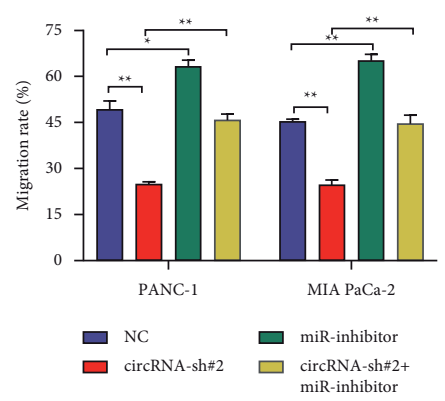

(d)

Figure 6: Continued. 


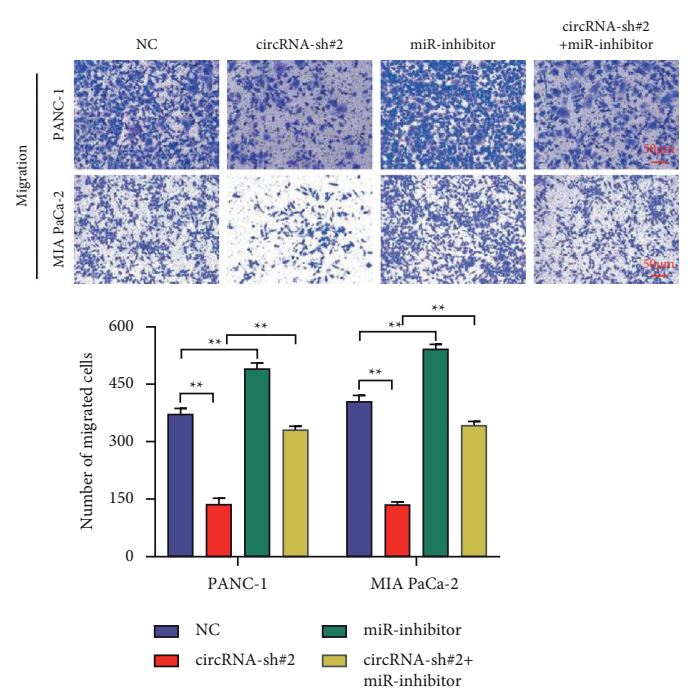

(e)

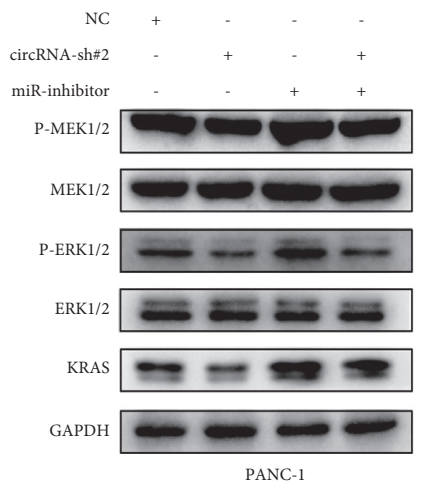

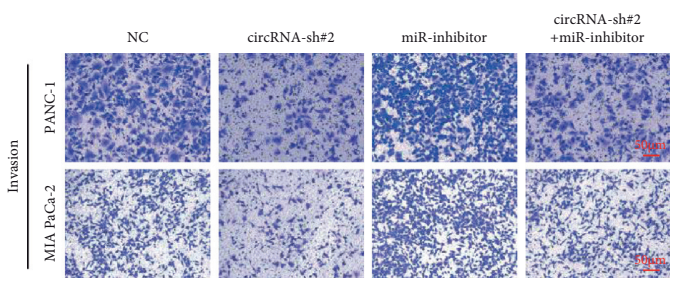

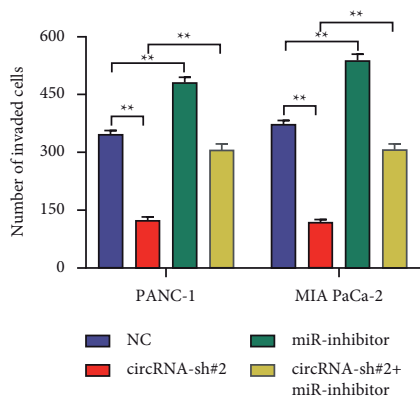

(f)

$(\mathrm{g})$

Figure 6: Circular RNA hsa_circ_0006117 facilitates pancreatic cancer (PC) development by adsorbing miR-96-5p. (a) The growth ability in circular RNA hsa_circ_0006117-silenced and (or) miR-96-5p-knockdown PC cells. (b) The colony-forming ability of PC cells in different processing groups. (c, d) The migratory ability of PC cells in different processing groups was verified by a wound healing assay. Representative images were obtained at $\times 40$ magnification (bars: $100 \mu \mathrm{m}$ ). (e, f) The invasive and migratory capabilities of PC cells were investigated by transwell migration (e) and invasion (f) assay, respectively. Representative images were obtained at $\times 200$ magnification (bars: $50 \mu \mathrm{m}$ ). (g) Protein expression of key genes in the MAPK signaling pathway in circular RNA hsa_circ_0006117-silenced and (or) miR96-5p-knockdown PC cells. All values were shown as means $\pm \mathrm{SD},{ }^{*} P<0.05$, and ${ }^{* *} P<0.001$.

another study [14] suggested that circ-PDE8A could facilitate the invasiveness capability of PC cells through the stimulation of the MET/ERK or AKT pathways, and exosomal circ-PDE8A has been related to the PC process and the prognosis of PC patients. In contrast, reduced circRNA expression has also been linked with the inhibition of PC progression. For example, Kong et al. [6] revealed that circNFIB1 regulates the miR-486-5p/PIK3R1 axis and further suppresses lymphatic metastasis in PC. Analogously, circular RNA hsa_circ_0006117 is reported to be low expressed and identified as a tumor-inhibiting factor in nonsmall cell lung carcinomas [27] and bladder cancer [28] recently. Surprisingly, our analysis results suggested that circular RNA hsa_circ_0006117 was highly expressed in PC, which indicated that it may have tissue specific and play a cancer-promoting role in PC. After all, the tissue specificity of circRNAs is one of their most striking features. However, current studies have not found a cancer-promoting role of circular RNA hsa_circ_0006117. We reported for the first time that circular RNA hsa_circ_0006117 is upregulated and played a promotive role in PC. These clues provided novel insights for further exploring the role of circular RNA hsa_circ_0006117. Furthermore, our results confirmed the PC tissue specificity of circular RNA hsa_circ_0006117 expression and revealed its independent biological function.

The KRAS/MAPK signaling pathway is strongly related to the growth and survival of cancer cells [29, 30]. This pathway is also involved in resistance to chemotherapy, autophagy, and metabolic reprogramming, all of which contribute to the malignant capacity of PC [19, 31]. In addition, KRAS promotes PC development through the regulation of noncoding RNA or nucleotide synthesis, while $K R A S$ is a well-known activator that mediates the phosphorylation/activation of the MAPK signaling pathway $[32,33]$. Here, our results also support this idea. However, whether there is an association between KRAS and circRNAs 


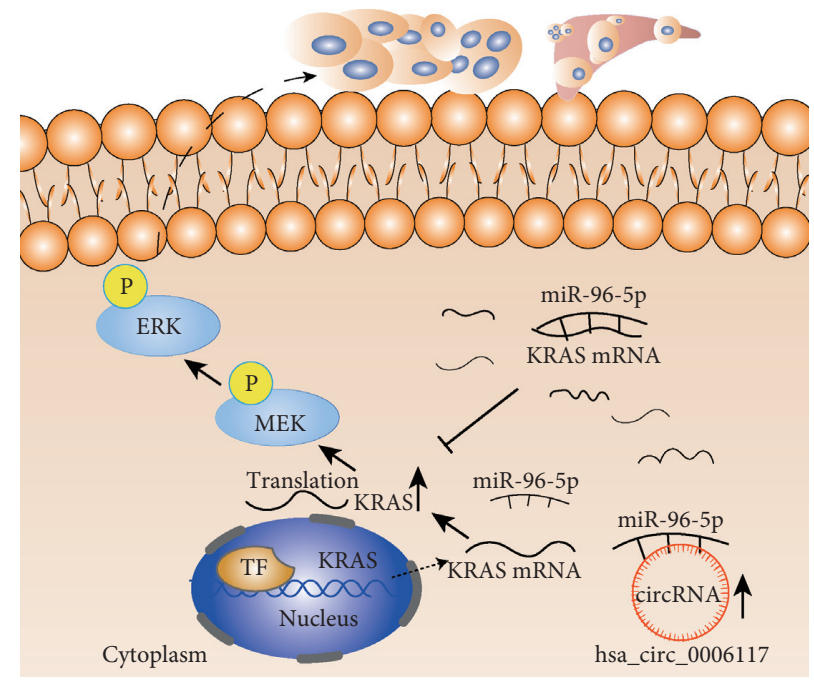

FiguRE 7: Schematic diagram of this research. Circular RNA hsa_circ_0006117 facilitates the malignant features of PC by regulating the miR-96-5p/KRAS/MAPK signaling pathway.

in PC has not been investigated. In our research, we first demonstrated that circular RNA hsa_circ_0006117 could upregulate the expression of KRAS via the competitive absorption of miRNA. And we found the cancer-promoting role of miR-96-5p in PC, which is consistent with previous work by others [34]. Intriguingly, previous studies [17, 35] have identified KRAS as a candidate for genetic therapy for PC treatment. Further, a recently published study suggested that an inhibitor targeting mutated KRAS represented an effective treatment for some types of tumors [36]. Besides, therapies targeting MEK/ERK are considered to be promising treatments to slow the progression of PC [31]. Our study indicated that circular RNA hsa_circ_0006117 could stimulate the MAPK signaling pathway via up-regulating the phosphorylation of MEK/ERK and accelerate PC progression in a KRAS-dependent manner. Combined, we believed that circular RNA hsa_circ_0006117 may be a promising pharmacological target, and exploring the functions of $K R A S$-associated circRNAs may be of value for clinical application.

Accumulating evidence has indicated that circRNAs can play both oncogenic and tumor-suppressor roles via sponging miRNAs [37, 38]. For instance, Luo et al. [39] uncovered that circCCDC9 modulates the miR-6792-3p/CAV1 axis, thereby suppressing the development of gastric cancer. Another study revealed [40] that hsa_circ_001783 adsorbed miR-200c-3p to accelerate the malignant behavior of breast cancer. In our study, we also discovered that circular RNA hsa_circ_0006117 plays an oncogenic role in PC by sponging a miRNA (miR-965 p). Notably, in similar research, most studies first identify miRNAs and then explore the associated downstream circRNA regulatory network. However, we first focused on the highly expressed genes in potential signaling pathways of PC and then searched for downstream targets and further determined the circRNA-related regulatory network, which led to miR-96-5p/KRAS and was identified as the downstream target of circular RNA hsa_circ_0006117 in PC. This provides a novel idea for the investigation of the ceRNA mechanism.
Of course, it is undeniable that not all ceRNA mechanisms can be successfully verified by this approach. Combined, all of our work indicated that circular RNA hsa_circ_0006117 promotes PC progression in a manner that is dependent on the downregulation of miR-96-5p.

\section{Conclusion}

In summary, we have identified circular RNA hsa_circ_0006117 as a specifically highly expressed circRNA in PC. We further found that circular RNA hsa_circ_0006117 facilitates the malignant behaviors of $\mathrm{PC}$ through regulating the miR-96-5p/KRAS/MAPK signaling pathway (Figure 7). These results suggest that circular RNA hsa_circ_0006117 may contribute to the potential therapeutic target of PC.

\section{Data Availability}

The data used to support the findings of this study are included within the article and supplementary information files.

\section{Conflicts of Interest}

The authors declare that there are no conflicts of interest regarding the publication of this study.

\section{Authors' Contributions}

Tao Liu and Zhiwei He led the current research and carried out bioinformatic analysis; Tao Liu and Lei Zhou carried out experiments and prepared the manuscript; Yankun Chen and Xueyi Jiang modified the manuscript and performed some supplementary experiments; Jian Xu contributed technical support and Jianxin Jiang provided funding support; all authors approved the final version of this article. 


\section{Acknowledgments}

This research was supported by the National Natural Science Foundation of China (Grant no. 81871965).

\section{Supplementary Materials}

Supplementary Figure S1: (A-B) the heat map obtained by R analysis showed the differentially expressed circRNAs in GSE69362 and GSE79634 datasets, respectively; (C) the relative mRNA content of circular RNA hsa_circ_0006117 and PTPRA in PC cells after actinomycin D treatment. All values were expressed as means $\pm S D$, ns: not significant, ${ }^{* *} P<0.001$. Supplementary Figure S2: the pattern diagram of circular RNA hsa_circ_0006117 small interfering RNA (siRNA). Supplementary Figure S3: (A) potential target genes (red) enriched on the MAPK signaling pathway; (B) potential target genes (red) enriched on the RAS signaling pathway; (C) differently expressed GRB2, IGF2BP2, and RAP1A from the GEPIA database; (D) the mRNA content of GRB2, IGF2BP2, and RAP1A in PC cells transfected with shRNA of circular RNA hsa_circ_0006117. All values were expressed as means $\pm \mathrm{SD},{ }^{*} P<0.05$. Supplementary Figure S4: (A-B) sequencing results of pmiR-RB-Report ${ }^{\mathrm{TM}}$ circular RNA hsa_circ_0006117-WT (A) and pmiR-RB-Report ${ }^{\text {TM }}$ circular RNA hsa_circ_0006117-MUT (B); (C-D) sequencing results of pmiR-RB-Report ${ }^{\mathrm{TM}} \mathrm{KRAS}-\mathrm{WT}(\mathrm{C})$ and pmiRRB-Report ${ }^{\mathrm{TM}}$ KRAS-MUT (D). (Supplementary Materials)

\section{References}

[1] R. L. Siegel, K. D. Miller, H. E. Fuchs, and A. Jemal, "Cancer statistics, 2021," CA: A Cancer Journal for Clinicians, vol. 71, no. 1, pp. 7-33, 2021.

[2] O. Strobel, J. Neoptolemos, D. Jäger, and M. W. Büchler, "Optimizing the outcomes of pancreatic cancer surgery," Nature Reviews Clinical Oncology, vol. 16, no. 1, pp. 11-26, 2019.

[3] E. Versteijne, J. A. Vogel, M. G. Besselink et al., "Meta-analysis comparing upfront surgery with neoadjuvant treatment in patients with resectable or borderline resectable pancreatic cancer," British Journal of Surgery, vol. 105, no. 8, pp. 946-958, 2018.

[4] M. Reyngold, E. M. O’Reilly, A. M. Varghese et al., "Association of ablative radiation therapy with survival among patients with inoperable pancreatic cancer," JAMA Oncology, vol. 7, no. 5, pp. 735-738, 2021.

[5] E. J. Buss, L. A. Kachnic, and D. P. Horowitz, "Radiotherapy for locally advanced pancreatic ductal adenocarcinoma," Seminars in Oncology, vol. 7754, no. 21, 2021.

[6] Y. Kong, Y. Li, Y. Luo et al., "CircNFIB1 inhibits lymphangiogenesis and lymphatic metastasis via the miR-486-5p/ PIK3R1/VEGF-C axis in pancreatic cancer," Molecular Cancer, vol. 19, no. 1, p. 82, 2020.

[7] S.-J. Deng, H.-Y. Chen, Z. Zeng et al., "Nutrient stress-dysregulated antisense lncRNA GLS-AS impairs GLS-mediated metabolism and represses pancreatic cancer progression," Cancer Research, vol. 79, no. 7, pp. 1398-1412, 2019.

[8] J. Yao, C. Zhang, Y. Chen, and S. Gao, "Downregulation of circular RNA circ-LDLRAD3 suppresses pancreatic cancer progression through miR-137-3p/PTN axis," Life Sciences, vol. 239, Article ID 116871, 2019.
[9] L. S. Kristensen, M. S. Andersen, L. V. W. Stagsted, K. K. Ebbesen, T. B. Hansen, and J. Kjems, "The biogenesis, biology and characterization of circular RNAs," Nature Reviews Genetics, vol. 20, no. 11, pp. 675-691, 2019.

[10] L. S. Kristensen, T. B. Hansen, M. T. Venø, and J. Kjems, "Circular RNAs in cancer: opportunities and challenges in the field," Oncogene, vol. 37, no. 5, pp. 555-565, 2018.

[11] X. Tang, H. Ren, M. Guo, J. Qian, Y. Yang, and C. Gu, "Review on circular RNAs and new insights into their roles in cancer," Computational and Structural Biotechnology Journal, vol. 19, pp. 910-928, 2021

[12] I. L. Patop, S. Wüst, and S. Kadener, "Past, present, and future of circRNAs," The EMBO Journal, vol. 38, Article ID e100836, 2019.

[13] Z. Wang, M. Deng, L. Chen et al., "Circular RNA circ-03955 promotes epithelial-mesenchymal transition in osteosarcoma by regulating miR-3662/metadherin pathway," Frontiers in Oncology, vol. 10, Article ID 545460, 2020.

[14] Z. Li, W. Yanfang, J. Li et al., "Tumor-released exosomal circular RNA PDE8A promotes invasive growth via the miR338/MACC1/MET pathway in pancreatic cancer," Cancer Letters, vol. 432, pp. 237-250, 2018.

[15] D. Uprety and A. A. Adjei, "KRAS: from undruggable to a druggable cancer target," Cancer Treatment Reviews, vol. 89, Article ID 102070, 2020.

[16] J. Cicenas, K. Kvederaviciute, I. Meskinyte, E. MeskinyteKausiliene, A. Skeberdyte, and J. Cicenas, "KRAS, TP53, CDKN2A, SMAD4, BRCA1, and BRCA2 mutations in pancreatic cancer," Cancers, vol. 9, no. 5, p. 42, 2017.

[17] A. M. Waters and C. J. Der, "KRAS: the critical driver and therapeutic target for pancreatic cancer," Cold Spring Harbor Perspectives in Medicine, vol. 8, no. 9, Article ID a031435, 2018.

[18] C.-M. Hu, S.-C. Tien, P.-K. Hsieh et al., "High glucose triggers nucleotide imbalance through O-GlcNAcylation of key enzymes and induces KRAS mutation in pancreatic cells," Cell Metabolism, vol. 29, no. 6, pp. 1334-1349, 2019.

[19] C.-S. Lee, L. C. Lee, T. L. Yuan et al., "MAP kinase and autophagy pathways cooperate to maintain RAS mutant cancer cell survival," Proceedings of the National Academy of Sciences, vol. 116, no. 10, pp. 4508-4517, 2019.

[20] C. Ding, G. Xi, G. Wang et al., "Exosomal circ-MEMO1 promotes the progression and aerobic glycolysis of non-small cell lung cancer through targeting MiR-101-3p/KRAS axis," Frontiers in Genetics, vol. 11, p. 962, 2020.

[21] K. Valencia, O. Erice, K. Kostyrko et al., "The Mir181ab1 cluster promotes KRAS-driven oncogenesis and progression in lung and pancreas," Journal of Clinical Investigation, vol. 130, no. 4, pp. 1879-1895, 2020.

[22] J. Chen, Y. Sun, Z. Ou et al., "Androgen receptor-regulated circFNTA activates KRAS signaling to promote bladder cancer invasion," EMBO Reports, vol. 21, Article ID e48467, 2020.

[23] M. A. Tempero, "NCCN guidelines updates: pancreatic cancer," Journal of the National Comprehensive Cancer Network, vol. 17, pp. 603-605, 2019.

[24] M. Takehara, Y. Sato, T. Kimura et al., "Cancer-associated adipocytes promote pancreatic cancer progression through SAA1 expression," Cancer Science, vol. 111, no. 8, pp. 2883-2894, 2020.

[25] M. F. C. Hansen, J. H. Storkholm, and C. P. Hansen, "The results of pancreatic operations after the implementation of multidisciplinary team conference (MDT): a quality 
improvement study," International Journal of Surgery, vol. 77, pp. 105-110, 2020.

[26] J. Li, Z. Li, P. Jiang et al., "Circular RNA IARS (circ-IARS) secreted by pancreatic cancer cells and located within exosomes regulates endothelial monolayer permeability to promote tumor metastasis," Journal of Experimental \& Clinical Cancer Research, vol. 37, no. 1, p. 177, 2018.

[27] S. Wei, Y. Zheng, Y. Jiang et al., "The circRNA circPTPRA suppresses epithelial-mesenchymal transitioning and metastasis of NSCLC cells by sponging miR-96-5p," EBioMedicine, vol. 44, pp. 182-193, 2019.

[28] F. Xie, C. Huang, F. Liu et al., "CircPTPRA blocks the recognition of RNA N6-methyladenosine through interacting with IGF2BP1 to suppress bladder cancer progression," Molecular Cancer, vol. 20, no. 1, p. 68, 2021.

[29] Q.-Y. Sun, L.-W. Ding, K. Johnson et al., "SOX7 regulates MAPK/ERK-BIM mediated apoptosis in cancer cells," Oncogene, vol. 38, no. 34, pp. 6196-6210, 2019.

[30] M. Burotto, V. L. Chiou, J.-M. Lee, and E. C. Kohn, "The MAPK pathway across different malignancies: a new perspective," Cancer, vol. 120, no. 22, pp. 3446-3456, 2014.

[31] K. M. Mann, H. Ying, J. Juan, N. A. Jenkins, and N. G. Copeland, "KRAS-related proteins in pancreatic cancer," Pharmacology \& Therapeutics, vol. 168, pp. 29-42, 2016.

[32] N. Santana-Codina, A. A. Roeth, Y. Zhang et al., "Oncogenic KRAS supports pancreatic cancer through regulation of nucleotide synthesis," Nature Communications, vol. 9, no. 1, p. 4945, 2018.

[33] Y. Liu, W. Feng, S. Gu et al., "The UCA1/KRAS axis promotes human pancreatic ductal adenocarcinoma stem cell properties and tumor growth," American Journal of Cancer Research, vol. 9, pp. 496-510, 2019.

[34] S. Yu, Z. Lu, C. Liu et al., "miRNA-96 suppresses KRAS and functions as a tumor suppressor gene in pancreatic cancer," Cancer Research, vol. 70, no. 14, pp. 6015-6025, 2010.

[35] S. Kamerkar, V. S. LeBleu, H. Sugimoto et al., "Exosomes facilitate therapeutic targeting of oncogenic KRAS in pancreatic cancer," Nature, vol. 546, no. 7659, pp. 498-503, 2017.

[36] D. S. Hong, M. G. Fakih, J. H. Strickler et al., "KRASG12C inhibition with sotorasib in advanced solid tumors," New England Journal of Medicine, vol. 383, no. 13, pp. 1207-1217, 2020.

[37] B. Chen and S. Huang, "Circular RNA: an emerging noncoding RNA as a regulator and biomarker in cancer," Cancer Letters, vol. 418, pp. 41-50, 2018.

[38] W. Huang, Y. Yang, J. Wu et al., "Circular RNA cESRP1 sensitises small cell lung cancer cells to chemotherapy by sponging miR-93-5p to inhibit TGF- $\beta$ signalling," Cell Death \& Differentiation, vol. 27, no. 5, pp. 1709-1727, 2020.

[39] Z. Luo, Z. Rong, J. Zhang et al., "Circular RNA circCCDC9 acts as a miR-6792-3p sponge to suppress the progression of gastric cancer through regulating CAV1 expression," Molecular Cancer, vol. 19, no. 1, p. 86, 2020.

[40] Z. Liu, Y. Zhou, G. Liang et al., "Circular RNA hsa_circ_ 001783 regulates breast cancer progression via sponging miR200c-3p," Cell Death \& Disease, vol. 10, no. 2, p. 55, 2019. 